\title{
Uncuffed Dialysis Catheter
}

National Cancer Institute

\section{Source}

National Cancer Institute. Uncuffed Dialysis Catheter. NCI Thesaurus. Code C122620.

A hemodialysis or peritoneal dialysis catheter that does not utilize a band-like structure at the exit site just under the skin to prevent slippage and infection. 